\title{
Proposal of a Reference Object in Dimensional Measurements using Computed Tomography
}

\author{
Amalija HORVATIĆ NOVAK*, Biserka RUNJE, Ivan SAMARDŽIĆ, Leon MAGLIĆ
}

\begin{abstract}
Computed tomography is a non-destructive method that uses the nature of X-ray in order to measure both inner and outer objects' geometry. Because of many advantages and possibilities of conducting both material analysis inspection and dimensional measurement in a non-destructive way, the method is increasingly represented in industry. However, the method is very complex and has a huge number of influence parameters that cause errors in measurement results. Consequently, the measurement uncertainty as well as metrological traceability in general case are not achieved. In order to minimize and eliminate systematic errors, the reference objects are used. The usage of reference object for the purpose of identification and compensation of systematic errors is a generally accepted approach to ensure traceability. This article gives an overview of existing reference objects used in dimensional metrology with computed tomography and presents a new reference object.
\end{abstract}

Keywords: computed tomography; dimensional measurement; metrological traceability; reference object; systematic error

\section{INTRODUCTION}

The method of dimensional measurement using computed tomography (CT) belongs to the group of 3D coordinate measurements. It is a method which enables measurement of both external and internal geometry of measured objects, by scanning objects using X-ray. The method was developed in the second half of the $20^{\text {th }}$ century by Godfrey Hounsfield and Allan Cormack and was initially used in Medicine. Since the middle of the 1980 s the method was used for non-destructive material analysis, while in the 2000s it found its application in dimensional measurements. The first industrial computed tomography scanner was introduced at a Control fair in Stuttgart, Germany by a company called Werth Messtechnick [1]. Fig. 1 shows the introduction of the method into different fields. Since then, many measuring equipment manufacturers offered different CT scanners, both industrial and metrological.

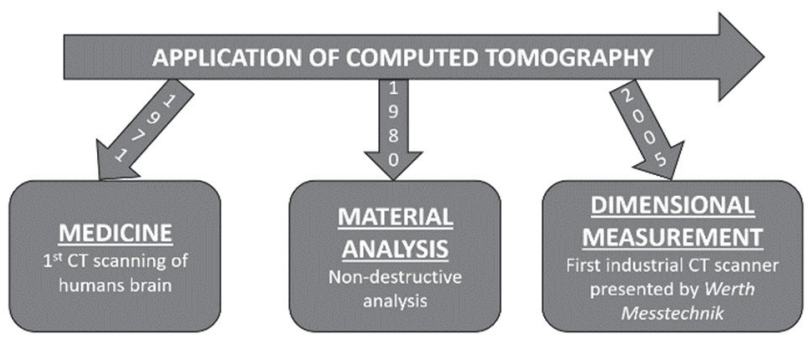

Figure 1 Application of computed tomography in various fields throughout history

The process of dimensional measurements using computed tomography is fairly complex, because of a large number of factors influencing the measurement process, resulting in systematic errors. In order to better understand the measurement process, influencing factors need to be identified and classified. Until today, many different classifications have been proposed. One of them is based on the step of measurement process which the factors influence. Such classification is further explained in [2]. Systematic errors are a consequence of dimensional and geometrical instability of the scanning process. The scanning process instabilities can include positioning of the measurement object with regard to the radiation source, object rotation during scanning, geometrical inconsistencies between the detector and radiation source, as well as geometrical changes of X-ray focus. Possible solutions for avoiding or reduction of systematic errors include: increasing geometrical and dimensional stability of the CT system and its components, improvement of reconstruction algorithms and application of reference objects [3]. Taking into account that users of computed tomography use existing CT systems with defined geometries and characteristics, as well as existing mathematical algorithms for reconstruction and analysis of data sets, the simple solution for solving systematic errors available to the end user is application of reference objects.

The paper gives an overview of the existing reference objects according to their purpose and, in accordance with the guidelines of the German VDI/VDE 2630 Part 3 standard, proposes a new reference object for CT dimensional measurements.

\section{MEASUREMENT TRACEABILITY}

Metrological traceability is defined as property of a measurement result whereby the result can be related to a reference through a documented unbroken chain of calibrations, each contributing to the measurement uncertainty [4]. Following the definition of metrological traceability, it is possible to achieve the assurance of traceability of measurement results by developing a reference object and by identifying the influencing parameters on the measurement system. This can further be achieved by estimating the influence of these parameters on measurement results, or in other words, by calculating measurement uncertainty. Metrological traceability chain is a sequence of measurement standards and calibration that is used to relate a measurement result to a reference [4]. Fig. 2 presents the metrological traceability chain for industrial computed tomography.

Considering the fact that computed tomography is a relatively new method used in dimensional metrology and taking account of the fact that there always exists a large number of influencing parameters within the measurement process, metrological traceability cannot be assured. However, many efforts have been made in order to assess 
measurement uncertainty as well as to achieve metrological traceability in CT dimensional measurements. Until today, measurement uncertainty in the field of dimensional measurements with computed tomography is best assessed according to ISO 15530-3 which involves the use of a reference object as similar as possible to measured object. Accordingly, in order to achieve metrological traceability and to evaluate measurement uncertainty in CT dimensional measurements, the reference object as similar as possible to the object of interest should be provided. In many cases it is impossible and unprofitable to provide another object with similar design, and for that reason it is necessary to provide a universal reference object which would eliminate as many systematic errors and which could be used in general case.

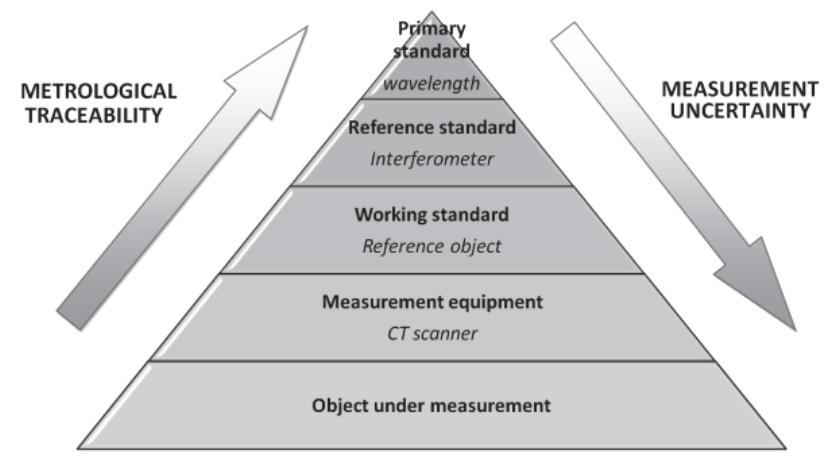

Figure 2 Measurement traceability pyramid for computed tomography

Using reference objects for identifying and compensation of systematic errors is a generally accepted approach for ensuring traceability by contact coordinate measurement machines (CMM's). Moreover, reference objects are frequently used for testing the performance of CMM's [5]. Since computed tomography belongs to the coordinate measurement methods, using reference objects is a logical way for determining possibilities of CT scanners and for ensuring metrological traceability.

\section{REFERENCE OBJECTS}

To ensure metrological traceability in CT dimensional measurements, a large number of objects have been proposed and investigated as possible reference objects. A scanning process depends on a huge number of input parameters i.e. scanning setup which is in direct connection to the objects' characteristics such as size and material density, resulting with systematic and random errors. Using a reference object allows correction of systematic shift, which is a consequence of systematic error, and brings the results closer to the actual value.

Considering the recognized use of reference objects in contact and optical three-coordinate methods, the original idea was to apply existing reference objects when measuring with industrial CT scanners [1]. However, the problem encountered is the excessive absorption of radiation, since such objects are usually made of steel. For this reason, some of the existing objects have been customized for use in computed tomography, but also a few new reference objects have been proposed. Fig. 3 shows some existing reference objects used for contact 3D measurements.

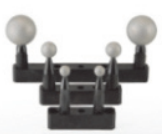

a)

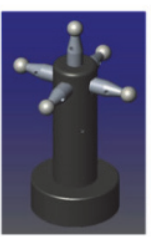

b)

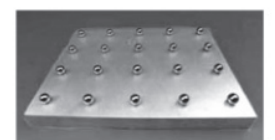

c)

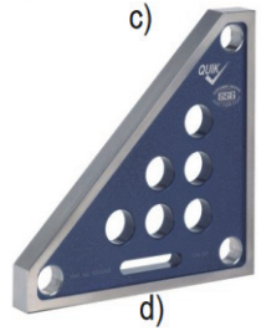

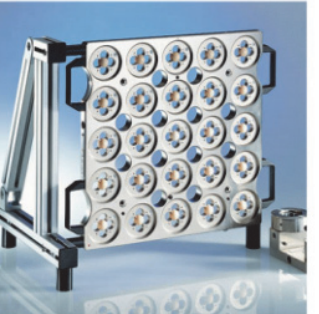

e)
Figure 3 Reference objects used in contact CMM: a) calibration balls [6]; b) star probe CMM [7]; c) steel ball plate [8]; d) QuikCheck gage [9]; e) ball plate [10]

Requirements on reference objects used in CT measurements are given with German standard VDI/VDE 2630Part 1.3 [11].

Depending on the purpose, reference objects in CT dimensional measurements can be divided into several groups:

a) Reference objects for scale error correction.

b) Reference objects for determination of threshold value.

c) Other.

\subsection{Reference Object for Scale Error Correction}

Reference objects with spheres are used for correction of voxel size, i.e. for scale error correction. Voxel size depends on object's position in CT scanner, i.e. on distance between X-ray source and detector as well as on sensor size on the detector. Scale error can occur as a result of heat generated in the radiation source, i.e. the impact of heat on the focus, causing focus shift. Other causes of scale error include geometrical errors of translational and rotational kinematic system of the CT scanner. Scale error causes significant systematic shift in measurement results and necessarily needs to be corrected. In order to correct the voxel size, unidirectional measurement characteristics are chosen. This type of dimensional characteristics is independent of defined boundaries between material and space i.e. independent of the chosen threshold value. In most cases, in order to correct voxel size, the distance between two or more sphere centres is used. Objects with ruby spheres, derived in the form of sticks, plates, tetrahedrons or hexahedrons, proved to be suitable for performing voxel size correction. The use of easily penetrable materials avoids the presence of beam hardening artefacts which influence measurement results. Fig. 4 shows some reference objects consisting of ruby spheres used for voxel size correction.

Besides the reference objects made of industrial ruby, objects of the same or similar geometries made of other materials are also used to correct voxel size. The examples of those objects are: dismountable alumina tetrahedral reference object and ball-bar made from ceramic balls on carbon fibre rod (Fig. 5). 


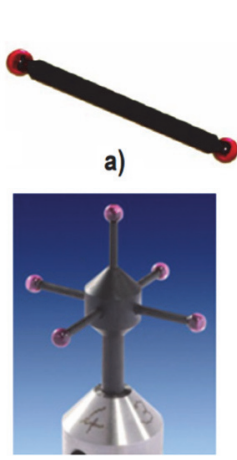

b)

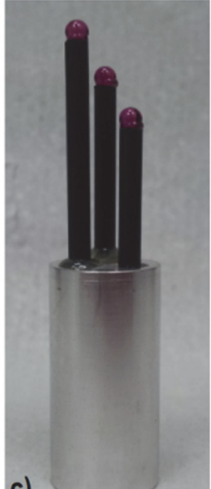

c)

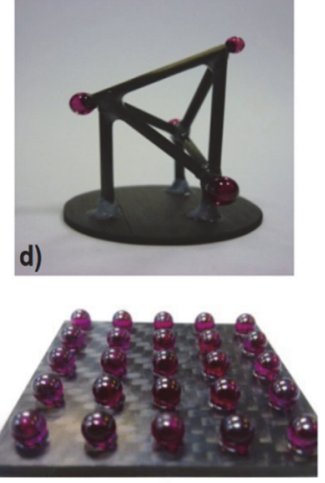

e)
Figure 4 Reference objects for scale error correction: a) carbon stick with ruby ball [12]; b) assembly of ruby spheres [1]; c) reference object with three ruby balls [13]; d) CT tetrahedron [14]; e) CT ball plate [15]

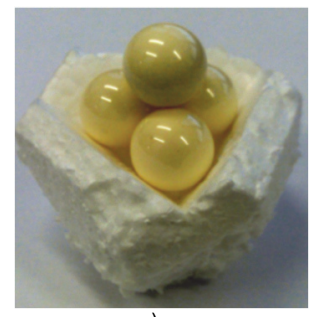

a)

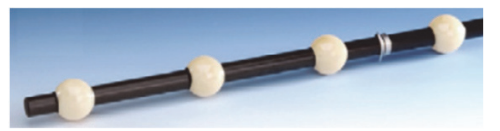

b)

Figure 5 Reference objects for scale error correction: a) dismountable alumina tetrahedral [16]; b) ball-bar [3]

Also, there were attempts to use reference objects made of higher density materials e.g. steel spheres. However, this approach has not been proven reliable due to presence of beam hardening artefacts [17].

The procedure for voxel size correction recommends scanning the reference object before and after scanning the object of interest. However, if the measuring system is such that reference object can be placed close to the object of interest in a way that both objects are captured in the same scanning, there is no need for the mentioned procedure of separate reference object scanning. Such approach significantly reduces the time required to conduct scale correction. Given the instability of the $\mathrm{X}$-ray source over a time period, voxel size correction is necessary for each measurement. Given the significance of the scale error in CT dimensional measurements, the procedure for scale correction should be included in the standard for application of industrial computed tomography for the purposes of dimensional measurements.

\subsection{Reference Objects for Determination of Threshold Value}

The second group of reference objects consists of objects with outer and inner measurement characteristics used for determination of threshold value. The process of selecting the optimal threshold value is achieved by repetitive measurements of both outer and inner measurands for different grey values $(g v)$. The optimal threshold value is the one at which differences (biases) between reference values and measured values of both outer and inner dimensional characteristics are as small as possible. This procedure for selecting threshold value was suggested by Carmignato et al. [18]. They use a reference object named fibre gauge, for the correction of a wrongly defined threshold value. The authors explain how wrongly defined threshold values affect measurement results. By defining threshold value lower than optimal, dimensions of inner characteristics will result with smaller dimensions than reference values, while dimensions of outer characteristics in that case will be larger. On the other hand, by defining threshold value higher than optimal, inner characteristics will result with larger dimensions than reference values, while dimensions of outer characteristics in that case will be smaller.

Proper selection of the threshold value is crucial in later edge detection, especially in case of bidirectional measurements, as shown in Fig. 6. For understanding the significance of proper threshold value determination, an example for distance $l$ is given in Fig. 6 .

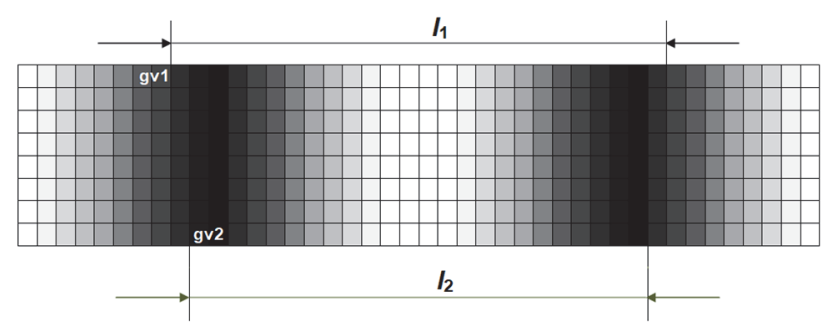

Figure 6 Distance / for two different grey values

Depending on the threshold value, the same distance can result with different amounts. When selecting threshold value of $g v_{1}$, distance between two surfaces will be equal to $l_{1}$, while in case when selecting threshold value of $g v_{2}$, the same distance will be equal to $l_{2}$. In order to minimize errors due to wrong threshold values, objects with both outer and inner dimensional characteristic are used. Some of them are shown in Fig. 7.

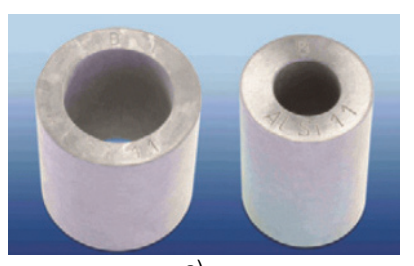

a)

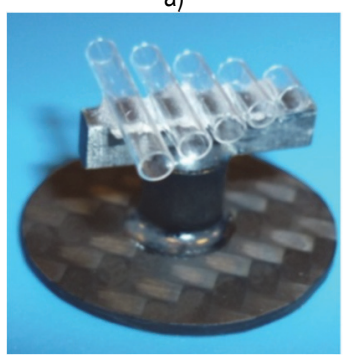

c)

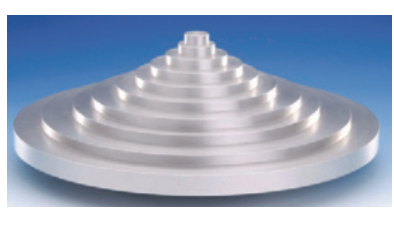

b)

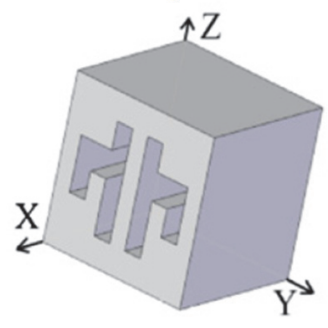

Figure 7 Reference objects for threshold determination: a) hollow cylinders [3]; b) step cylinder [3]; c) pan flute gauge [14]; d) cactus gauge [19]

Following the guidelines given in [11], it is preferable that reference object for threshold determination is made from the same or similar material and has a similar size and shape as the object of interest. In that case defined threshold value can be reliably applied for the object of interest as well [18]. 


\subsection{Other Reference Objects}

Besides mentioned reference objects, objects with different geometries and purposes are developed and investigated. It is possible to underline the step shaped objects, of whether prismatic (Fig. 5a, 5c, 5d) or cylindric form (Fig. 5b). Step shaped reference objects are used in order to investigate maximum possible objects' thickness to be radiated with the available X-ray source. Also, step reference objects were used to investigate influence of object's orientation during the scanning process [20] and for determination of beam hardening software correction. Some examples of step reference objects are given in Fig. 8.

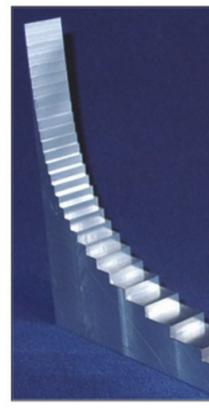

a)

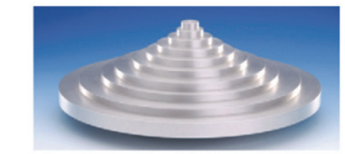

b)

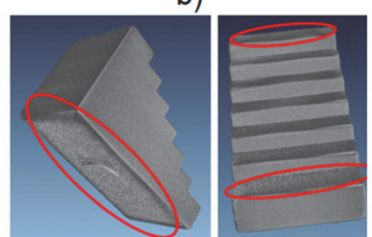

c)

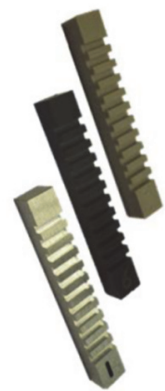

d)
Figure 8 Step reference objects:a) step wedge [21]; b) step cylinder without central hole [3]; c) step workpiece [20]; d) step gauge [22]

Possibilities of CT measurement system in a specific case are tested by using either aluminium hexahedron with complex geometry (Fig. 9a), or hole plate (Fig. 9b) available in two sizes, smaller one made from steel and bigger one from aluminium [23]. Furthermore, QFM cylinder (Fig. 9c) is used for CT validation held as a part of international comparison on CT dimensional metrology, called CT audit [24]. Fig. 9 shows other reference objects.

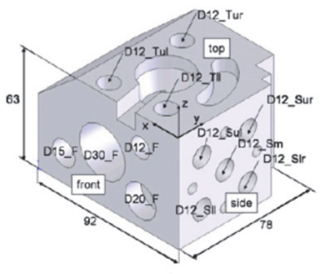

a)

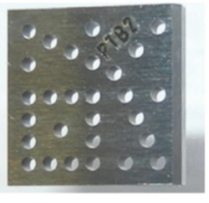

b)

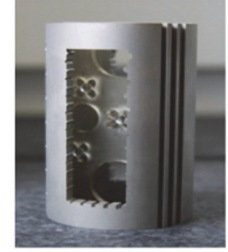

c)
Figure 9 Other reference objects: a) aluminium hexahedron [25]; b) hole plate [26]; c) QFM cylinder [14]

Manufactures of CT scanners sometimes state that metrological traceability can be assured by using reference objects. The most commonly mentioned method for achieving metrological traceability is the use of so-called calibration rods/sticks, which, with a certain measurement uncertainty, are used for voxel size correction. However, the voxel size correction is only one of the contributions in achieving metrological traceability. Furthermore, some of the device manufacturers define maximum permissible error (MPE) by conducting many distance measurements between ruby spheres on reference objects. It is important to emphasize that declared MPE value does not represent maximum permissible error of the device in general case.
Given the lack of procedures for CT dimensional measurements and the fact that there is a huge number of parameters that influence measurement system and result in either systematic or random errors, the use of reference objects in order to eliminate as many systematic errors is necessary. Until today there are a large number of patented and used objects, with the aim to eliminate systematic errors or with the purpose to check possibilities of measurement system. However, there is not one universal reference object which will allow qualification and adjustment of measurement system, as well as elimination of systematic errors in general case. For this reason, a new reference object is proposed.

\section{REQUIREMENTS ON REFERENCE OBJECTS}

An overview of the available reference objects in CT dimensional measurements is given. None of the available objects eliminates all systematic errors in general case. The reason for this lies in the extremely broad purpose and capabilities of CT scanners that allow analysis of objects of different materials, sizes and geometrical complexity. In order to suggest new reference object of a wider purpose, guidelines and suggestions on objects size, shape and performance need to be followed.

According to the guidelines given in [11], reference object should have the following properties:

- Material used for reference object must be stated by all means.

- The surface roughness of the features to be probed should be negligibly small.

- Irregularity of form should be negligibly small.

- Measurement uncertainty of results shall be significantly smaller than the MPE of the CMM used for reference measurements.

- The material and dimensions of the reference object should be as similar as possible to the object of interest. Besides guidelines given in VDI/VDE 2630 Part 1.3, some other suggestions, following previous experience and citations from the literature, are given:

- Reference object should have features in which the results of dimensional measurements are independent of chosen threshold value [21].

- Reference object should be made of material which is possible to radiate with available X-ray source [3].

- Properties of material used for reference object should be unchangeable during the time [3].

- The reference object must be visible on the detector in its entirety, also when larger geometric magnifications [21].

- Material used for reference objects fixtures should have low density and should not influence the attenuation of the reference object [27].

\section{A NEW REFERENCE OBJECT}

Following the guidelines given in German standard VDI/VDE 2630 Part 1.3 and instructions available from previous research, a new reference object is proposed. The object consists of three groups of spheres, differing in size and material. The choice of material is left to the choice of each individual user according to its needs. Here, proposed object consists of aluminium, ruby and ceramic spheres 
interconnected with easily penetrable carbon rods. Tab. 1 shows material properties with indicative values for the selected material groups. The data is available at online database [28].

Reference object consists of carbon rods oriented in three vertical axes, each carrying ruby and aluminium spheres. The centre of the object is the largest of all spheres, ceramic sphere. In total, the object consists of thirteen spheres. Fig. 10a shows the proposed reference object.

The central sphere is full, while ruby and aluminium spheres contain central holes. Sphere diameters, as well as distances between spheres, are defined in proportions given with the smallest suggested diameter i.e. diameter of aluminium sphere $(R)$.

Besides the reference object, the fixture is proposed (Fig. 10b). Following suggestions which recommend low density material for fixtures, easily penetrable carbon is proposed. Previous researches recommend slightly tilted position of the objects in regard to X-ray radiation source $[3,29,30]$. Therefore, the fixture should be designed and made in such a way to assure slightly tilted orientation of the object during the scanning process. By choosing the appropriate orientation of the object in the scanning process, it is possible to improve the image quality and finally to reduce the measurement uncertainty of the measurement results.

Table 1 Material properties [28]

\begin{tabular}{|l|c|c|c|c|c|}
\hline \multicolumn{2}{|c|}{} & \multicolumn{3}{c|}{ Spheres } & \multicolumn{2}{c|}{ Rods } \\
\cline { 3 - 5 } & & Aluminium alloy & Industrial ruby & $\begin{array}{c}\text { Zirconium oxide } \\
\text { ceramics }\end{array}$ & $\begin{array}{c}\text { Carbon fibre reinforced } \\
\text { polymer composite }\end{array}$ \\
\hline Material density & $\mathrm{g} / \mathrm{cm}^{3}$ & $2,70-2,84$ & $3,92-4,00$ & $6,00-6,13$ & $1,55-1,58$ \\
\hline Young's modulus of elasticity & $\mathrm{GPa}$ & $\sim 70$ & 390410 & $195-205$ & $9,8-10,2$ \\
\hline Temperature expansion coefficient & $10^{-6} \mathrm{~K}^{-1}$ & $21,8-26,1$ & $8,7-9,1$ & $129-154$ \\
\hline Melting point & ${ }^{\circ} \mathrm{C}$ & $568-657$ & $2000-2100$ & $-(0,4)-0,2$ & $2550-2700$ \\
\hline Thickness & $\mathrm{HV}$ & $35-150$ & $1830-2030$ & $1330-1470$ & $3652-3697$ \\
\hline
\end{tabular}

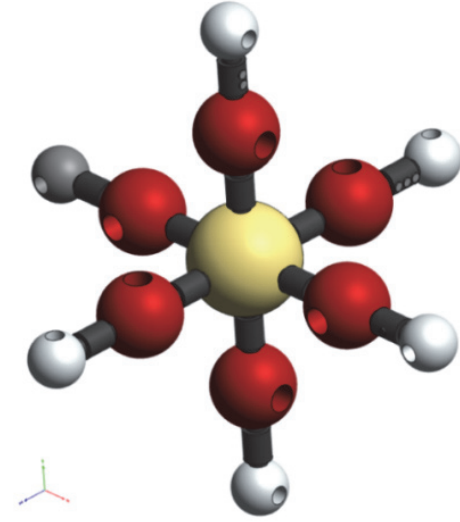

a)

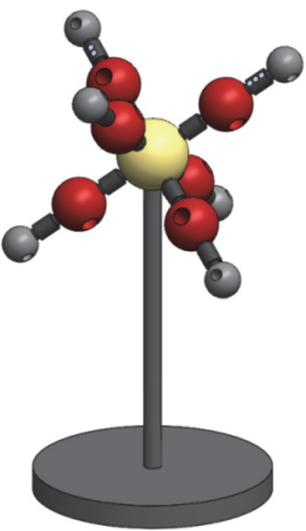

b)
Figure 10 Proposed reference object a) without fixture; b) with fixture

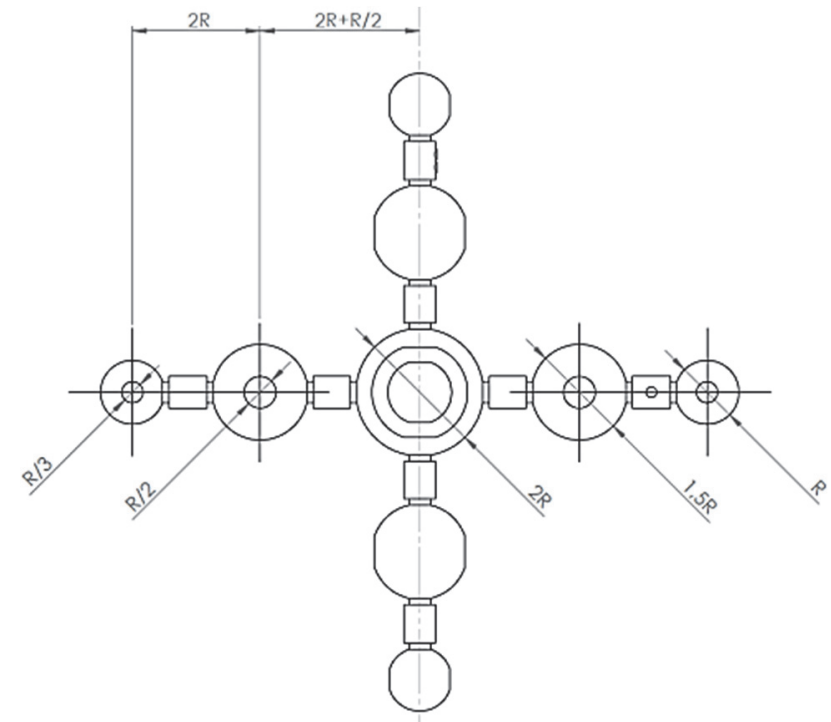

Figure 11 Drawing of proposed reference object

Fig. 11 shows relations between feature dimensions. According to the drawing, the distance between aluminium and ruby spheres is equal to the sum of two diameters of the smallest sphere, while the distance between the ruby spheres and the ceramic sphere is increased by the amount of radius of the smallest sphere in order to achieve the same length of all carbon fibre rods. The smallest radius is the one of aluminium spheres.

Ruby spheres are 1,5 times bigger than aluminium spheres, while ceramic sphere is 2 times bigger than aluminium sphere. Also, the holes' diameters are 3 times smaller than the outer diameter of carrying spheres. With this type of approach, a linear increase in the dimension of characteristic shapes (here spheres) is achieved, as recommended by the guidelines given in [11].

Since the proposed object is symmetrical along all three axes and the nominal values of defined diameters are the same, each axis should be marked somehow. In order to distinguish between axes, on proposed reference object each rod is marked with one, two or three dots. Marking the rods in this way does not affect measurement results.

\section{CONCLUSION}

In the process of measuring with computed tomography there are many systematic and random errors as a result of large number of influencing parameters. Systematic error of measurement or simply systematic error is defined as component of measurement error that in replicate measurements remains constant or varies in a predictable manner [4]. In order to eliminate systematic errors, a correction of measurement result with the aim to compensate for a known systematic error can be applied. The most significant systematic errors in industrial computed tomography are scale errors and errors due to wrongly selected threshold value. Correction of those errors can be successfully conducted with the use of different reference objects.

In the paper, a proposal of a new reference object for the purpose of elimination of systematic errors is given. The new reference object can be used for voxel size correction by using distances between different sphere 
centres. Since the spheres are placed in three different axes, correction can be made for each axis separately using one distance or by combining three distances, one for each axis, for scale correction of $3 \mathrm{D}$ coordinate system in whole. Also, distances between spheres on different axes and between spheres made of different material can be used.

Furthermore, the correction of optimal threshold value can be applied by monitoring the relations in dimensions between inner and outer sphere diameter. The proper selection of threshold value can be made by simultaneously measuring outer and inner diameter and choosing that threshold value for which deviations between reference and measured values are minimal. Since the object consists of spheres made from material with different absorption coefficient, the same (one) object can be used when measuring different materials or multi-material objects. In the proposed object, ruby, aluminium and ceramic are chosen for sphere materials, but selection of sphere materials can be made regarding the users' needs, but with respect to guidelines given in German standard VDI/VDE 2630 Part 1.3.

Considering that the size of the reference object is not defined by the amount, but with regard to the smallest sphere diameter, the object can be made in different size versions. With such proposal of the object, a linear increase in the dimension of characteristic shapes (here spheres) is achieved. Smaller versions of the object are suggested for higher geometrical magnifications, while bigger versions should be used for smaller geometrical magnifications.

\section{REFERENCES}

[1] Kruth, J. P., Bartscher, M., Carmignato, S., Schmitt, R., De Chiffre, L., \& Weckenmann, A. (2011). Computed tomography for dimensional metrology. Cirp AnnalsManufacturing Technology, 60(2), 821-842. https://doi.org./10.1016/j.cirp.2011.05.006

[2] Horvatić Novak, A., Runje, B., \& Stepanić, J. (2016). Capabilities of industrial computed tomography in the field of dimensional measurements. Advances in Production Engineering \& Management, 12(3), 245-253. https://doi.org/10.14743/apem2017.3.255

[3] Bartscher, M, Hilpert, U., Goebbels, J., \& Weidemann, G. (2007). Enhancement and proof of accuracy of industrial computed tomography (CT) measurements. Cirp AnnalsManufacturing Technology, 56(1), 495-498. https://doi.org/10.1016/j.cirp.2007.05.118

[4] JCGM 200:2012 (2012). International vocabulary of metrology - Basic and general concepts and associated terms (VIM).

[5] Štrbac, B., Balazs, M., Rodić, D., Nagy, J., \& Hadžistević, M. (2020). Analysis of Characteristics of Non-Commercial Software Systems for Assessing Flatness Error by Means of Minimum Zone Method. Tehnicki vjesnik - Technical Gazette, 27(2), 535-541. https://doi.org./10.17559/TV-20190603084835

[6] See http://www.eht.com.my/webshaper/pcm/pictures/Koni ca\%20Minolta/Range\%207/reliablepict003.jpg

[7] See https://www.precisionballs.com/images/pm-star-75A_ 000.jpg

[8] See https://www.precisionballs.com/images/ball plate1.jpg

[9] See http://www.auto-met.com/gsgage/images/QuikChek8.jpg

[10] See https://www.retter.de/images/kugelplatte/kugelplatte-36 $0-1 . j p g$

[11] VDI/VDE 2630 Part 1.3 (2011). Computed tomography in dimensional measurement: Guideline for the application of DIN EN ISO 10360 for coordinate measuring machines with
CT sensors.

[12] Lim, S. H. (2013, July). Automated 3D CT: Industrial developments and trends. $2^{\text {nd }}$ Singapore International NDT Conference \& Exhibition, Singapore.

[13] Lifton, J. J., Cross, K. J., Malcolm, A. A. \& McBride, J. W. (2013, April). A reference workpiece for voxel size correction in X-ray computed tomography. Presented at the International Conference on Optics in Precision Engineering and Nanotechnology - icOPEN2013, Singapore. https://doi.org/10.1117/12.2020916

[14] Carmignato, S. \& Pierobon, A. (2011, June). Preliminary Results of the "CT Audit" Project: First International Intercomparison of Computed Tomography Systems for Dimensional Metrology. Paper presented at the International Symposium on Digital Industrial Radiology and Computed Tomography, Berlin, Germany.

[15] Müller, P., Hiller, J., Cantatore, A., Tosello, G., \& De Chiffre, L. (2012, June). New reference object for metrological performance testing of industrial CT systems. Paper presented at the $12^{\text {th }}$ Euspen International Conference, Stockholm, Sweden.

[16] Leonard, F., Brown, S. B., Withers, P. J., Mummery, P. M., \& McCarthy, M. B. (2014). A new method of performance verification for x-ray computed tomography measurements. Measurement Science \& Technology, 25(6). https://doi.org/10.1088/0957-0233/25/6/065401

[17] Andersen, L. \& Holmberg, M. (2016, February).An investigation into $C T$ uncertainty using a high-density reference object. Paper presented at the $6^{\text {th }}$ International Conference on Industrial Computed Tomography - iCT, Wels, Austria.

[18] Carmignato, S., Dreossi, D., Mancini, L., Marinello, F., Tromba, G., \& Savio, E. (2009). Testing of x-ray microtomography systems using a traceable geometrical standard. Measurement Science \& Technology, 20(8). https://doi.org/10.1088/0957-0233/20/8/084021

[19] Kiekens, K., Welkenhuyzen, F., Tan, Y., Bleys, P., Voet, A., Kruth, J. P., \& Dewulf, W. (2011). A test object with parallel grooves for calibration and accuracy assessment of industrial computed tomography (CT) metrology. Measurement Science \& Technology, 22(11). https://doi.org/10.1088/0957-0233/22/11/115502

[20] Welkenhuyzen, F., Kiekens, K., Pierlet, M., Dewulf, W., Bleys, P., Kruth, J. P., \& Voet, A. (2009, May). Industrial Computer Tomography for Dimensional Metrology: Overview of Influence Factors and Improvement Strategies. Paper presented at the $4^{\text {th }}$ International Conference on Optical Measurement Techniques for Structures \& Systems, Antwerpen, Belgium.

[21] Müller, P. (2010). Use of reference objects for correction of measuring errors in X-ray computed tomography. Department of Mechanical Engineering at Technical University of Denmark.

[22] De Chiffre, L., Andreasen, J. L., Christensen, L. B., Feidenhans'l, R. K., Vinter, B., Jensen, N. T., Arentoft, M., Jørgensen, P. B., Vester-Christensen, M., \& Schmidt, P. R. (2014). Centre for Industrial Application of CT scanning (CIA-CT) - Four years of results 2009-2013. Department of Mechanical Engineering at Technical University of Denmark.

[23] Bartscher, M., Sato, O., Härtig, F., \& Neuschaefer-Rube, U. (2014). Current state of standardization in the field of dimensional computed tomography. Measurement Science \& Technology, 25(6). https://doi.org/10.1088/0957-0233/25/6/064013

[24] Carmignato, S., Pierobon, A., \& Savio, E. (2012). CT Audit: Interlaboratory Comparison of Computed Tomography Systems for Dimensional Metrology, Final Report. Laboratory of Industrial and Geometrical Metrology, Padova.

[25] Schmitt, R. \& Niggemann, C. (2010). Uncertainty in measurement for x-ray-computed tomography using calibrated 
work pieces. Measurement Science \& Technology, 21. https://doi.org/10.1088/0957-0233/21/5/054008

[26] Kasperl, S., Schielein, R., Sukowski, F., Hornberger, P., \& Gruber, A. (2014, October). CT simulation study to demonstrate material impact using hole plates. Paper presented at the $11^{\text {th }}$ European Conference on NonDestructive Testing - ECNDT, Prague, Czech.

[27] Müller, P., Cantatore, A., Andreasen, J. L., Hiller, J., \& De Chiffre, L. (2013). Computed tomography as a tool for tolerance verification of industrial parts. Procedia CIRP, 10, 125-132. https://doi.org/10.1016/j.procir.2013.08.022

[28] Material property data. See http://www.matweb.com/

[29] Weckenmann, A. \& Krämer, P. (2010). Predetermination of Measurement Uncertainty in the Application of Computed Tomography. Product Lifecycle Management: Geometric Variations, 317-330. https://doi.org/10.1002/9781118557921.ch17

[30] Cantatore A. \& Müller P. (2011). Introduction to computed tomography. Technical University of Denmark, Department of Mechanical Engineering.

\section{Contact information:}

Amalija HORVATIĆ NOVAK, PhD, Postdoctoral researcher (Corresponding author)

University of Zagreb,

Faculty of Mechanical Engineering and Naval Architecture, Ivana Lučića 5, 10000 Zagreb, Croatia

E-mail: amalija.horvatic@fsb.hr

Biserka RUNJE, PhD, Full professor

University of Zagreb,

Faculty of Mechanical Engineering and Naval Architecture,

Ivana Lučića 5, 10000 Zagreb, Croatia

E-mail: biserka.runje@fsb.hr

Ivan SAMARDŽıĆ, PhD, Full professor

University of Slavonski Brod

Mechanical Engineering Faculty,

Trg Ivane Brlić Mažuranić 2, 35000 Slavonski Brod, Croatia

E-mail: isamardzic@unisb.hr

Leon MAGLIĆ, PhD, Full professor

University of Slavonski Brod,

Mechanical Engineering Faculty,

Trg Ivane Brlić Mažuranić 2, 35000 Slavonski Brod, Croatia

E-mail: Imaglic@unisb.hr 